\title{
Ulfat, Fahimah / Ghandour, Ali (Hg.) (2020). Islamische Bildungsarbeit in der Schule. Theologische und didaktische Überlegungen zum Umgang mit ausgewählten Themen im Islamischen Religionsunterricht. Wiesbaden: Springer VS. ISBN 978-3-658-26720-9. 3 I 6 Seiten.
}

\section{Mehmet H. Tuna}

Universität Innsbruck (mehmet.tuna@uibk.ac.at)

Der vorliegende Sammelband "Islamische Bildungsarbeit in der Schule“ widmet sich der Aufgabe, ausgewählte Themen, wie etwa Tod, Propheten-Erzählungen, Schöpfungserzählung, Hadith und Sira, Umgang mit dem Koran und der Koranexegese, der Theodizee u. a. im Hinblick auf ihren Bildungsgehalt, die didaktische Relevanz sowie Transfermöglichkeiten zu reflektieren. Das Buch umfasst insgesamt 12 Beiträge, bei denen es sich - bis auf wenige Fälle - im Kern um theologische Auseinandersetzungen handelt, die thematisch gereiht wurden.

Die ersten Beiträge setzen sich mit der vielfältigen Bedeutung und Auslegung überlieferter Texte, insbesondere mit dem Koran, auseinander. Jameleddine Ben Abdeljelil widmet sich der koranischen Deutung der Begriffe kufr und kāfirūn (dt. Unglaube und Ungläubige) sowie den innermuslimischen takfirrDiskursen (1-30) und der damit verbundenen Frage, inwiefern religiöse, voneinander abweichende Traditionen im Islam legitim sind oder Formen von Unglauben darstellen. Der Beitrag von Ali Ghandour (31-48) beschäftigt sich mit der „Hypothetizität der muslimischen normativen Tradition.“ Ghandour führt die Hypothetizität, ar-Rāzī folgend, darauf zurück, dass „Texte nur ein hypothetisches Wissen hergeben können“ (37). Darin sieht der Autor zum einen die innerislamische Pluralität begründet und zum anderen die Möglichkeit, Lernende zum Umgang mit religiösen Traditionen und Texten zu befähigen.

Diesen Auseinandersetzungen folgen Beiträge, die sich mit dem Offenbarungsverständnis und der Anthropologie auseinandersetzen. So beschäftigt sich Fahimah Ulfat mit den koranischen Erzählungen sowie den $t a ' w \bar{\imath} l$ - bzw. 'išărî-Auslegungsformen (49-64) und konstatiert, dass das Deuten und Aufdecken der latenten Bedeutung(en) von Koranversen nur durch „,[d]as Verständnis vom Koran als Urnarrativ und Anstoß einer unabgeschlossenen Kette mündlicher Kommunikation“ (57) ermöglicht werden kann. Im anschließenden Beitrag geht Florian A. G. Lützen (65-94) auf das islamische Verständnis des Herzens (qalb) und seiner Funktion in Zusammenspiel mit der Ratio ( $a q l$ ) und der Triebseele (nafs) des Menschen ein.

Anschließend folgen Beiträge zu den Prophetenerzählungen und ihrem Bildungsgehalt. So führt Ruggero Vimercati Sanseverino (95-144) in seinem Beitrag zum Umgang mit dem Propheten Muhammad im Sinne einer erfahrungsbezogenen Prophetologie in das Themenfeld der Propheten ein. Er diskutiert die Thematisierung von Propheten im Religionsunterricht aus der "Sicht der theologischen Forschung“ (99). Diesem folgen weitere Beiträge von Alexander Schmidt (146-164) zu Adam sowie von Hamida Sarah Behr (167-188) zu Maria und Jesus.

Die nächsten Beiträge beschäftigen sich mit dem Einsatz und der Nutzung grafischer und audiovisueller Medien, wie etwa Figuren, Karikaturen oder Videospielen im Islamischen Religionsunterricht (IRU). Bilal Badat beleuchtet in seinem Aufsatz diverse „Muslimische Standpunkte zur figürlichen Darstellung“ (189-212) und Daniel Roters diskutiert in seinem Beitrag, welche religiös bedeutsamen Narrative Videospiele beinhalten können (213-248). 
Den Abschluss markieren Beiträge, die sich mit dem Leiden in der Welt auseinandersetzen. Lejla Demiri zeigt in ihrem Aufsatz "Eine Theologie des Todes“, wie muslimisch-theologische Konzepte von Tod und Vergänglichkeit „zu einem besseren Verständnis des Menschseins“ beitragen können (149-264). Demiri zufolge fördert „[d]ie Erinnerung an den Tod“ Demut und bietet „die Möglichkeit, sich selbst auf dem spirituellen Pfad zum Schöpfer zu transzendieren“ (262). Dara Alani (265-288) wiederum arbeitet in ihrem Aufsatz verschiedene Zugänge muslimischer als auch nicht-muslimischer Denker*innen, Mystiker und Philosophen zum Thema des Bösen und Üblen im Leben aus und sieht dieses Thema als eine gute Möglichkeit, „,mit Kindern oder insbesondere mit Jugendlichen in ein echtes Gespräch über Glauben und Religion einzutreten“ (286). Den Abschluss bildet der Beitrag von Timothy Winter (289311), der sich insbesondere mit den mu'tazilītischen, aš 'arītischen und māturīdischen Diskursen zum „Leiden von Tieren, Kindern und unschuldigen Erwachsenen“ auseinandersetzt. Hervorgehoben wird unter anderem das muslimische Verständnis vom Leiden als „,eine Form göttlicher Pädagogik oder Therapie.“ „[D]as Unglück führt die Gläubigen auf den Pfad zur Erlösung“, so Winter (294).

Die vorgestellten Beiträge von Ben Abdeljelil, Ghandour, Ulfat, Lützen, Sanseverino, Schmidt, Behr, Badat, Roters und Alani weisen insofern strukturelle Ähnlichkeiten auf, als sie den fachtheologischen Auseinandersetzungen religionspädagogische, pädagogische sowie didaktische Überlegungen und / oder „Hinweise für die Weiterarbeit“ (siehe etwa Ben Abdeljelil: 22-26) folgen lassen. Die Beiträge von Demiri und Winter wiederum begnügen sich mit der theologischen Auseinandersetzung und gehen auf die religionspädagogische und -didaktische Bedeutung ihrer Ausarbeitungen nicht explizit ein.

Insgesamt kommen die religionspädagogischen und -didaktischen Überlegungen und Hinweise einem Versuch gleich, eine Brücke zwischen der Islamischen Theologie und der Islamischen Religionspädagogik und -didaktik zu schlagen.

Das darin liegende Potenzial, Themen aus der verschränkten Perspektive zweier Disziplinen - hier der Islamischen Theologie und Religionspädagogik/-didaktik - zu reflektieren, wird aber nur in Teilen ausgeschöpft, denn zum einen setzen sich die Autor*innen nachvollziehbarerweise mit den Themen primär aus der Theologie bzw. Theorie der eigenen Fachdisziplin heraus auseinander, zum anderen bleiben die religionspädagogischen und -didaktischen Überlegungen in vielen Beiträgen knapp und relativ abstrakt.

Für die Lesenden bzw. Lehrenden, die in den religionspädagogischen und -didaktischen Überlegungen mitunter direkt adressiert werden, würde eine gleichwertige Verschränkung von Theologie und Religionspädagogik die in den Beiträgen vorgestellten Überlegungen und Ansätze wesentlich nachvollziehbarer machen und überdies einen ganzheitlichen Zugang zur Islamischen Bildung ermöglichen. 